deficiency syndrome (AIDS) and others concerned in the AIDS industry.

There seems, however, to be some muddled thinking about this matter. A life insurance policy is not another aspect of state funded social security but a straightforward financial transaction. In the past it was understood that an application for an insurance policy, even for a few hundred pounds, would necessitate a full medical examination. I some life shortening condition was found it was equally understood that cover might be refused or accepted only on increased terms. I assume that a detailed medical examination has been found to be uneconomical for smaller policies, but, presumably, this does not imply the right to defraud the company.

In discussing the test for antibodies to the human immunodeficiency virus (HIV), some counsellors have been suggesting that the subject should seek (perhaps additional) life insurance before the blood test is performed. This is surely no more ethical than advising an overweight 40 year old man with polydipsia to increase his life insurance before his fasting blood glucose concentration is investigated.

Life insurance companies owe it to their shareholders and policyholders (particularly those holding "with profit" policies) to take all possible care to exclude poor risk lives-for whatever reason. We keep hearing of the "rights" of various groups in our society. A carrier of HIV antibodies has every right to advice, civilised handling all times, and, when necessary, full medical treatment. He or she does not have the right to cheat insurance companies.

Hillingdon Hospital,

Middlesex UB8 3NN

\section{Infantile apnoea and home monitoring}

SIR,-Dr Peter G F Swift's letter (11 July, p 118) about infantile apnoea and home monitoring clearly reflects his belief in the value of alarms. We agree that alarms may be useful in selected patients (and parents), but some points need clarification. Children have died while being monitored despite the alarm responding, ${ }^{1}$ and, as Dr Swift says, this must be explained to the parents. Though Dr Swift has encountered no deaths during home monitoring so far, Emery et al have already pointed out that it will still take over 100 years to prove scientifically whether apnoea alarms save lives. ${ }^{2}$

Dr D M Reid (p 119) correctly reminds us that for parents scientific proof is not the only issue. Nevertheless, Dr Swift's statement that monitors "helped many parents to sleep, reduced over anxiety, and improved parent-child interaction" must be challenged, particularly as five families subsequently declined a second monitor. No monitor is trouble free, and comprehensive suppor arrangements must be arranged. Our own experience of 70 monitored children (32 siblings and 38 infants who had experienced severe "apparent life threatening events") showed that almost half of the parents needed to contact the hospital at some time during monitoring for mechanical or medical problems. ${ }^{3}$ This was despite frequent outpatient and telephone contact, especially during the first weeks of monitoring.

The importance of the National Institutes of Health consensus report is, as Professor Hamish Simpson (30 May, p 1367) states, that for the first time the use of apnoea alarms is considered for other conditions than sudden infant death syndrome. ${ }^{4}$ We welcome this as we believe that it is necessary to analyse the reasons why an alarm is being used. Attacks of lifelessness requiring vigorous or mouth to mouth resuscitation in infants who have suffered severe apparent life threatening events have a high risk of recurrence (40\%) and probably of causing sudden death. ${ }^{56}$ All such infants should thus receive a home apnoea alarm.

In our experience, however, most parents of siblings of victims of the sudden infant death syndrome do not need a home alarm. The risks to a sibling have probably been exaggerated in the past. ${ }^{7}$ The syndrome and the use of monitors are fully explained and any parental worries dealt with during pregnancy and before discharge. The child is reviewed regularly, especially around the time of the previous infant's death, and parents of such infants may contact the hospital if they are anxious.

If the parents cannot cope or sleep because of their fear of the sudden infant death syndrome despite our reassurance they are given a monitor. The lack of scientific proof should never deny such parents an alarm, for their sake as much as for the child's. Home monitors are not, however, the perfect answer, and yet many parents now feel guilty if they do not use an alarm "just in case something happens." Dr Swift, in stating that "it is surprising that many paediatricians are reluctant to provide respiratory monitors for use at home," puts similar pressure on paediatricians. Clinical judgment and the lack of local finances for monitors and follow up may mean that the correct decision is not to provide an alarm. The clearest message from the National Institutes of Health is that, despite the difficulties, it is time for scientific evaluation of the monitors and psychological problems related to tham so that the correct decisions may be made, based on fact not emotion.

K P DUNNE

Rotunda Hospital Dublin

T G MATTHEWS

1 Dunne KP, Matthews TG. Apnoea monitors and sudden infant death Arch Dis Child 1985;60:688.

2 Emery JL, Waite AJ, Limerick SR, Carpenter RG. Apnoea monitoring and cot deaths. Arch Dis Child 1987;62:99.

Dunne KP, Matthews TG. Home monitoring and SIDS. Practical problems. $I M \mathcal{J}$ (in press).

4 National Institutes of Health. Consensus statement of development conference on infantile apnoea and home monitoring. Sept 29-Oct 1 1986. Pediatrics 1987;79:292-9.

5 Dunne KP, Matthews TG. Clinical presentation and manage ment of "near miss" SIDS. Pediatrics 1987;79:889-93.

6 Oren J, Kelly D, Shannon DC. Identification of a high risk group for SIDS among infants who were resuscitated for sleep apnoea. Pediarrics 1986;77:495-9.

7 Irgeens LM, Skjaerven R, Peterson DR. Prospective assessment of recurrent risk in sudden infant death syndrome siblings. f Pediatr 1984;104:349-51.

SIR,-I agree with many of the points raised by Dr Peter G F Swift (11 July, p 118) regarding the possible benefits of home apnoea monitoring in siblings of victims of the sudden infant death syndrome and infants who have suffered previous apnoeic attacks.

Since 1980 we have monitored 288 infants ( 244 siblings of victims and 44 infants with apnoea) using the monitor described by Dr Swift. Like him, we have found that most families have found monitoring helpful: $28 \%$ of the families in which one child has died as a result of the syndrome have requested a second monitor and six families have used a third. Only three families to date have declined a second monitor.

Several points regarding the issuing of home monitors merit further emphasis. It must be explained clearly to all parents that there is absolutely no guarantee of infant survival. In our experience, most parents can accept this. Parents should be shown how to use the monitor, and possible advantages and disadvantages should be clearly explained, preferably before a final decision about monitoring is made. If monitoring is to be undertaken all parents should be instructed in basic resuscitation techniques, and there must be an adequate 24 hour back up service to deal with any problems that may arise.
I would, however, disagree with Dr Swift on one point. There is at least one recorded case, ${ }^{1}$ and several anecdotal cases, of death occurring while an infant was being monitored. In our experience this has occurred only once to date, in a sibling of a victim of the sudden infant death syndrome. These parents are currently using a monitor for a subseyuent infant and do not in any way regard the monitor as a failure because their previous infant died.

Finally, I would agree wholeheartedly with the comments of Dr David M Reid (11 July, p 119) to the effect that some of the benefits of monitoring may not be easy to assess in scientific publications. I suspect that those who have not lost a child in this way cannot fully appreciate the difficulties faced by parents in handling another baby. In the absence of any investigation that might assure parents that a second infant will not suffer the same fate as its sibling, constant observation is deemed necessary. In my view, if such parents think that an apnoea monitor will help them in caring for their baby one should be provided-that is, the decision should eventually be made by the parents in full knowledge of the facts. I would, however, emphasise that no parents should be talked into using a monitor or be made to feel guilty if they decline one, and the continuing use of a monitor should be seriously questioned if the monitor itself seems to be causing problems.

Yorkhill Cot Death Unit,

ANN HaRvie

Royal Heath Unit,

Glasgow G3 8S J

1 Lewak $M$. Sudden infant death syndrome in a hospitalised infant on an apnoea monitor. Pediatrics 1975;56:196-8.

\section{The myelodysplastic syndromes}

SIR,-Drs D $M$ Layton and $G \quad J$ Mufti (25 July, p 227) draw attention to the main features of the myelodysplastic syndromes and the need for further research into new therapeutic strategies. The problem, of course, is that we have only a very limited understanding of the disease processes, which makes our therapeutic choices difficult. It is now generally accepted that the syndromes arise from a premalignant clonal abnormality of a haemopoietic stem cell ${ }^{1}$ and that the various clinical manifestations reflect a multistage progression to overt leukaemia.

Treatment may be directed towards suppression or elimination of the abnormal cells or, as Drs Layton and Mufti suggest, to differentiation of the functionally immature preleukaemic cells. It is perhaps worth drawing attention to the potential attractions of tretinoin (retinoic acid) in the management of these conditions. While retinoic acid is well established as a differentiating agent for preleukaemic cell lines in culture,,$^{23}$ there is an additional rationale for its use. It has been known for some time that retinoids prevent chemically induced skin cancer in mice and other tumours, possibly by interfering with tumour promotion. Retinoic acid can cause regression of experimental premalignant lesions ${ }^{45}$ and suppress radiation induced leukaemia in mice, though it is not clear whether this is due to immune stimulation or to a direct effect on the leukaemic cells. ${ }^{6}$ A recent randomised therapeutic trial of isotretinoin (13-cis retinoic acid) compared with supportive treatment alone showed that in non-sideroblastic patients with less than $6 \%$ blasts in the marrow there was a significant increase in survival at one year in the group who received treatment from $36 \%$ to $77 \%$.

This is encouraging, though it remains to be confirmed, but we still have little information about the mode of action or whether there is a longer term benefit in suppression of abnormal 